\title{
IncRNA-NEF is downregulated in osteosarcoma and inhibits cancer cell migration and invasion by downregulating miRNA-21
}

\author{
QINGLEI YANG ${ }^{1,2}$, HONGYING YU ${ }^{3}$, QINGSHUI YIN ${ }^{1,4}$, XIAOMING HU ${ }^{1}$ and CHENGCHENG ZHANG ${ }^{1}$ \\ ${ }^{1}$ Department of Orthopedics, Guangzhou School of Clinical Medicine, Southern Medical University, Guangzhou, \\ Guangdong 510515; Departments of ${ }^{2}$ Orthopedics and ${ }^{3}$ Pharmacy, Yuebei People's Hospital, Shaoguan, Guangdong 512000; \\ ${ }^{4}$ Department of Orthopedics, Guangzhou General Hospital of Guangzhou Military Command (Liuhuaqiao Hospital), \\ Guangzhou, Guangdong 510515, P.R. China
}

Received September 8, 2018; Accepted March 15, 2019

DOI: $10.3892 / \mathrm{ol} .2019 .10276$

\begin{abstract}
Long non-coding RNA (lncRNA)-NEF inhibits cancer metastasis in hepatocellular carcinoma; however, its role in other malignancies is unknown. The present study revealed that plasma IncRNA-NEF was downregulated, while miRNA-21 was upregulated in patients with osteosarcoma compared with healthy controls. Plasma lncRNA-NEF and miRNA-21 were negatively correlated in patients with osteosarcoma and healthy controls. Downregulation of lncRNA-NEF and upregulation of miRNA-21 distinguished patients with osteosarcoma from healthy controls. IncRNA-NEF overexpression mediated the inhibition of miRNA-21 expression in osteosarcoma cell lines, while miRNA-21 overexpression did not significantly affect the expression of lncRNA-NEF. lncRNA-NEF overexpression inhibited, while miRNA-21 overexpression promoted, migration and invasion of osteosarcoma cell lines in vitro. miRNA-21 overexpression partially compensated the inhibitory effects of IncRNA-NEF overexpression on osteosarcoma cell migration and invasion. Therefore, lncRNA-NEF may inhibit cancer cell migration and invasion in osteosarcoma by downregulating miRNA-21.
\end{abstract}

\section{Introduction}

Osteosarcoma is a rare type of malignancy which originates in bone and affects $\sim 5$ in 1,000,000 patients (1). In spite of the low incidence, osteosarcoma has a high mortality rate due to the high prevalence rate of metastases by the time of first diagnosis (2). Improved treatment strategies have increased the survival time of patients with osteosarcoma $(3,4)$. However,

Correspondence to: Dr Qingshui Yin, Department of Orthopedics, Guangzhou School of Clinical Medicine, Southern Medical University, 1023 Shatainan Road, Guangzhou, Guangdong 510515, P.R. China

E-mail: tbqrn07@163.com

Key words: osteosarcoma, long non-coding RNA-NEF, miRNA-21, invasion, migration the overall five-year survival rate of patients with this disease remains $\sim 30 \%$ (5). The mechanisms underlying the pathogenesis of osteosarcoma have not been fully elucidated, potentially hindering the development of novel treatment strategies for this disease (6). Therefore, in-depth studies on the molecular mechanisms involved in osteosarcoma may improve its treatment.

In addition to protein-coding genes, the human genome contains a large number of genes which are transcribed to non-coding RNAs, including microRNAs (miRNAs) and long non-coding RNAs (lncRNAs) (7,8). miRNAs and lncRNAs participate in the regulation of cell growth, proliferation, differentiation, migration and invasion by interacting with a number of signaling pathways $(7,8)$. Previous studies have revealed that non-coding RNAs serve key roles in a number of human diseases, including metabolic diseases, heart diseases and cancer $(9,10)$. As an oncogenic miRNA, miRNA-21 promotes tumor development in different types of cancer, including osteosarcoma (11). miRNA-21 may participate in tumorigenesis through interactions with lncRNA (12). lncRNA neighboring enhancer of FOXA2 (IncRNA-NEF) is a recently identified tumor suppressor lncRNA which is involved in hepatocellular carcinoma (13). To the best of our knowledge, no studies investigating the interaction between lncRNA-NEF and miRNAs have been reported. The present study revealed that IncRNA-NEF may inhibit cancer cell migration and invasion in osteosarcoma by downregulating miRNA-21.

\section{Materials and methods}

Plasma specimens. Blood specimens $(5 \mathrm{ml})$ were collected from 49 patients with osteosarcoma and 42 healthy volunteers who were admitted to Guangzhou General Hospital of Guangzhou Military Command (Guangzhou, China) between September 2015 and June 2018. Blood was placed in anticoagulation tubes and centrifuged at room temperature for $15 \mathrm{~min}$ at $2,000 \mathrm{x} g$ to collect the plasma. The inclusion criteria of patients with osteosarcoma were as follows: i) Osteosarcoma was diagnosed by pathological examinations; ii) osteosarcoma stage I or II; iii) patients who were diagnosed and treated for the first time; and iv) patients who were willing to participate in the study. The exclusion criteria were as follows: i) Presence 
of co-morbidities; and ii) patients who were treated for other clinical disorders in the three months prior to admission. The patient group included 26 males and 23 females, with an age range of $12-33$ years and a mean age of $19.3 \pm 4.2$ years. The control group was composed of 23 males and 19 females, with an age range of 13-30 years and a mean age of 18.8 \pm 4.7 years. The two groups had similar gender and age distributions. Ethical approval was obtained from the Ethics Committee of Guangzhou General Hospital of Guangzhou Military Command. The study followed the tenets of the Declaration of Helsinki, and written informed consent was obtained from all patients and controls after the nature and possible consequences of the study were explained.

Cell culture. The human osteosarcoma cell lines MG-63 and U2OS were used in the current study. The two cell lines were purchased from the American Type Culture Collection (ATCC; Manassas, VA, USA). Eagle's Minimum Essential Medium (cat no. 30-2003; ATCC) supplemented with $10 \%$ heat-inactivated fetal bovine serum (FBS; Sangon Biotech Co., Ltd., Shanghai, China) was used to cultivate cells in an incubator $\left(37^{\circ} \mathrm{C}, 5 \% \mathrm{CO}_{2}\right)$.

Reverse transcription-quantitative polymerase chain reaction $(R T-q P C R)$. Total RNA was extracted from plasma and cells using TRIzol ${ }^{\circledR}$ reagent (Invitrogen; Thermo Fisher Scientific, Inc., Waltham, MA, USA) and miRNAs were extracted from plasma and cells using the miRNeasy Mini kit (Qiagen GmBH, Hilden, Germany), according to the manufacturer's protocol. An Applied Biosystems ${ }^{\mathrm{TM}}$ High-Capacity cDNA Reverse Transcription kit (Applied Biosystems; Thermo Fisher Scientific, Inc.) and a miScript II RT kit (Qiagen GmBH) were used for reverse transcription. qScript One-Step RT-qPCR kit (Quanta Bisciences, Inc., Beverly, MA, USA) and mirVana qRT-PCR miRNA Detection kit (Thermo Fisher Scientific, Inc.) were used to perform qPCR reactions in an ABI 7500 System (Applied Biosystems; Theremo Fisher Scientific Inc.). The miRNA-21 primer pair was purchased from Sigma-Aldrich (cat. no. MIRAP00047; Merck KGaA, Darmstadt, Germany). The following primer pairs and the miRNA-21 primers were used for the qPCR: IncRNA-NEF forward, 5'-CTGCCGTCT TAAACCAACCC-3' and reverse, 5'-GCCCAAACAGCTCCT CAATT-3'; $\beta$-actin forward, 5'-GACCTCTATGCCAACACA GT-3' and reverse, 5'-AGTACTTGCGCTCAGGAGGA-3'; U6 forward, 5'-CTCGCTTCGGCAGCACA3' and reverse, 5'-AAC GCTTCACGAATTTGCGT-3'. The aforementioned primers were designed and synthesized by Shangai GenePharma Co., Ltd. (Shanghai, China). The following thermocycling conditions were used for the qPCR: Initial denaturation at $95^{\circ} \mathrm{C}$ for $55 \mathrm{sec}, 40$ cycles of $95^{\circ} \mathrm{C}$ for $22 \mathrm{sec}$ and $60^{\circ} \mathrm{C}$ for $44 \mathrm{sec}$. mRNA levels were quantified using the $2^{-\Delta \Delta \mathrm{Cq}}$ method (14). miRNA-21 expression was normalized to U6, while IncRNA-NEF was normalized to $\beta$-actin.

Vectors, miRNAs and cell transfection. MISSION ${ }^{\circledR}$ microRNA Mimic hsa-miR-21* (cat no. HMI0372) and MISSION ${ }^{\circledR}$ miRNA, Negative Control 1 (cat no. HM0002) were purchased from Sigma-Aldrich; Merck KGaA. IncRNA-NEF expression pIRES vectors and empty pIRES vectors were purchased from Shanghai GenePharma Co., Ltd. IncRNA-NEF sequence refers to a previous study (13). Lipofectamine ${ }^{\circledR} 2000$ (Invitrogen; Thermo Fisher Scientific, Inc.) was used to transfect $5 \times 10^{5}$ cells with miRNA $(50 \mathrm{mM})$ and vectors $(15 \mathrm{mM})$. Transfection was performed according to the manufacturer's protocol. Untransfected cells were used as control (C) cells. Cells transfected with MISSION ${ }^{\circledR}$ miRNA, Negative Control 1 or empty vectors were used as negative control (NC) cells. Transfection efficacy was determined by RT-qPCR. Cells were harvested $24 \mathrm{~h}$ following transfection for subsequent experimentation.

Transwell migration and invasion assay. Cell migration and invasion were quantified when IncRNA-NEF and miRNA overexpression rates reached $200-250 \%$ following $24 \mathrm{~h}$ transfection. Cultrex ${ }^{\circledR}$ 96-well cell migration/invasion chambers $(8 \mu \mathrm{m})$ were purchased from Bio-Techne (Minneapolis, $\mathrm{MN}$, USA). The assays were performed according to manufacturer's protocol. Briefly, serum-free Eagle's Minimum Essential Medium was used to prepare single cell suspensions $\left(5 \times 10^{4}\right.$ cells $/ \mathrm{ml}$ ). A total of $5 \times 10^{3}$ cells in $100 \mu \mathrm{l}$ were transferred to the upper chamber of each well, and the lower chamber was filled with Eagle's Minimum Essential Medium supplemented with $20 \%$ FBS. Cells were maintained in an incubator $\left(37^{\circ} \mathrm{C}\right.$, $5 \% \mathrm{CO}_{2}$ ) for $2 \mathrm{~h}$. Membranes were subsequently stained with $0.5 \%$ crystal violet (Sigma-Aldrich; Merck KGaA) at $25^{\circ} \mathrm{C}$ for $20 \mathrm{~min}$. The invasion assay was performed in the same way as the migration assay; however, the upper chamber was pre-coated with Matrigel (cat no. 356234; Millipore; Merck $\mathrm{KGaA}$ ) at room temperature for $6 \mathrm{~h}$ prior to experimentation.

Statistical analysis. All experiments were repeated three times and data are presented as the mean \pm standard deviation. All statistical analyses were performed using GraphPad Prism 6 software (GraphPad Software Inc., La Jolla, CA, USA). Correlations between plasma lncRNA-NEF and miRNA-21 were analyzed by Pearson's correlation test. Receiver operating characteristic (ROC) curve analysis was performed using patients with osteosarcoma as true positive cases and healthy controls as true negative cases. Comparisons between two groups were performed using the Student's t-test and comparisons among multiple groups were performed by a one-way analysis of variance followed by the Tukey test. $\mathrm{P}<0.05$ was considered to indicate a statistically significant difference.

\section{Results}

Plasma levels of IncRNA-NEF and miRNA-21 are altered in patients with osteosarcoma compared with healthy controls. RT-qPCR results revealed that, compared with the healthy control group, plasma levels of lncRNA-NEF were significantly decreased in patients with osteosarcoma $(\mathrm{P}<0.05$; Fig. 1A). By contrast, plasma levels of miRNA-21 were significantly increased in patients with osteosarcoma compared with healthy controls $(\mathrm{P}<0.05$; Fig. 1B). These data suggest that lncRNA-NEF and miRNA-21 may be implicated in osteosarcoma.

Altered plasma levels of IncRNA-NEF and miRNA-21 distinguish patients with osteosarcoma from healthy controls. To evaluate the diagnostic value of IncRNA-NEF and miRNA-21 for osteosarcoma, ROC curve analysis was 
A

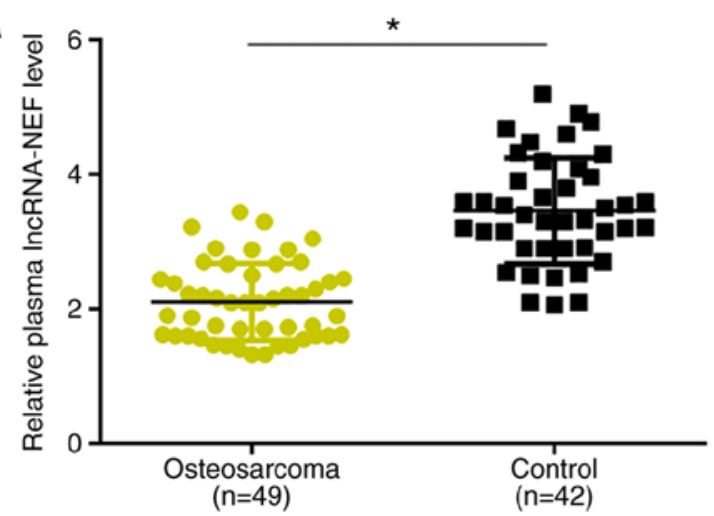

B

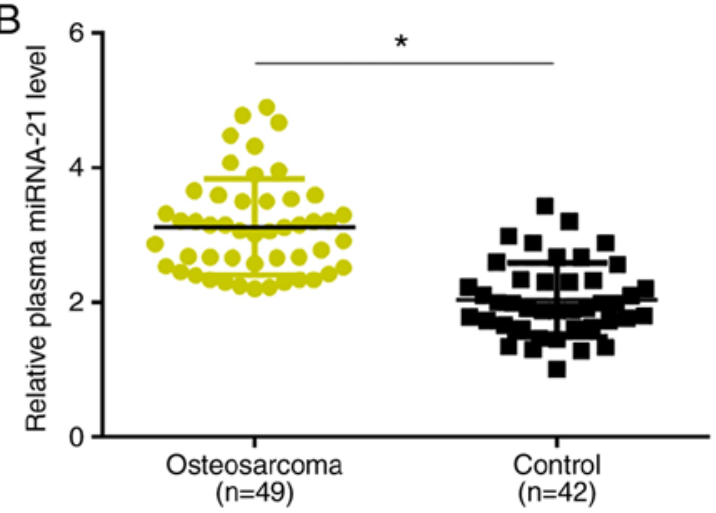

Figure 1. Plasma levels of lncRNA-NEF and miRNA-21 are altered in patients with osteosarcoma compared with healthy controls. (A) Plasma levels of lncRNA-NEF were significantly decreased in patients with osteosarcoma compared with healthy controls. (B) Plasma levels of miRNA-21 were significantly increased in patients with osteosarcoma compared with healthy controls. " $\mathrm{P}<0.05$. miRNA, micro RNA; IncRNA, long non-coding RNA; NEF, neighboring enhancer of FOXA2.
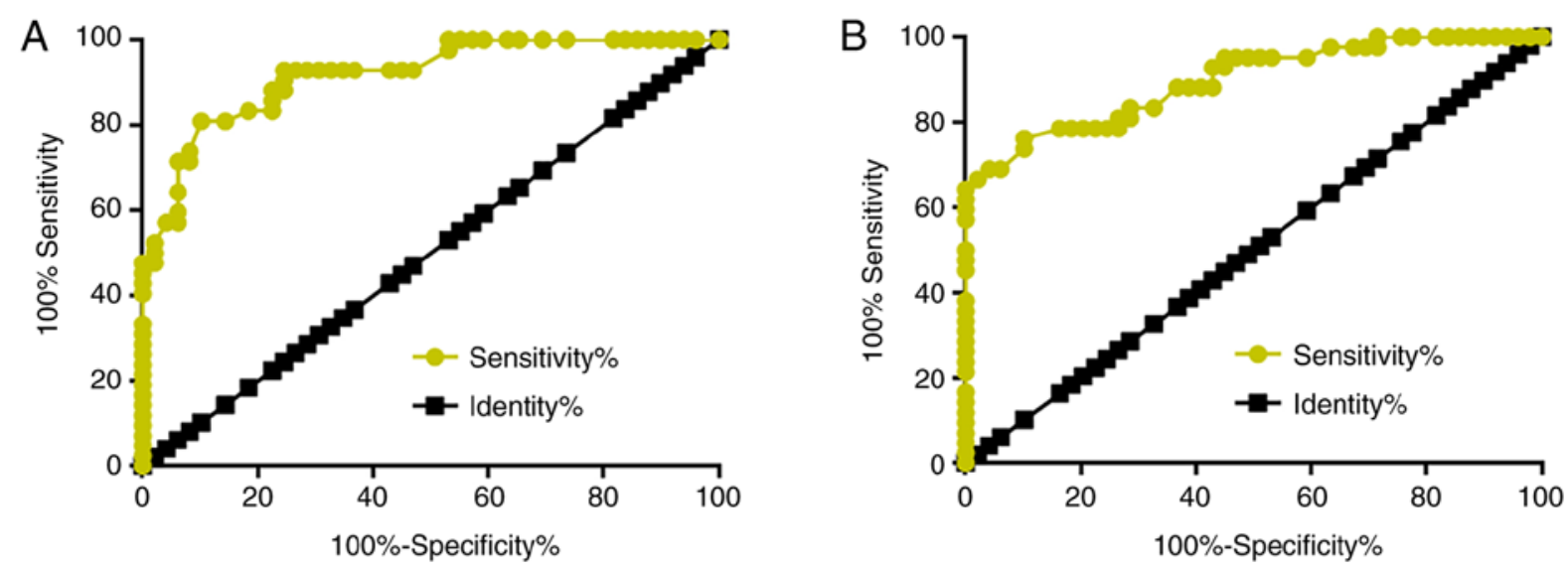

Figure 2. Altered plasma levels of lncRNA-NEF and miRNA-21 distinguish patients with osteosarcoma from healthy controls. Receiver operating characteristic curve analysis revealed that (A) downregulation of lncRNA-NEF and (B) upregulation of miRNA-21 distinguished patients with osteosarcoma from healthy controls. miRNA, micro RNA; lncRNA, long non-coding RNA; NEF, neighboring enhancer of FOXA2.

performed with patients with osteosarcoma as true positive cases and healthy controls as true negative cases. For plasma lncRNA-NEF, the area under the curve (AUC) was 0.9176, with a standard error of 0.02793 and $95 \%$ confidence interval (CI) of $0.8629-0.9724$ (Fig. 2A). For plasma miRNA-21, the AUC was 0.9009 , with a standard error of 0.02863 and $95 \% \mathrm{CI}$ of 0.8457-0.9662 (Fig. 2B).

Plasma lncRNA-NEF and miRNA-21 are significantly and reversely correlated in both patients with osteosarcoma and healthy controls. Correlations between plasma lncRNA-NEF and miRNA-21 in patients with osteosarcoma and healthy controls were analyzed by the Pearson's correlation test. Results revealed that plasma levels of IncRNA-NEF and miRNA-21 were significantly and reversely correlated in patients with osteosarcoma $(\mathrm{P}<0.05$; Fig. 3A). In addition, a significantly reverse correlation between plasma levels of lncRNA-NEF and miRNA-21 was demonstrated in healthy controls $(\mathrm{P}<0.05$; Fig. 3B).

IncRNA-NEF overexpression leads to inhibited miRNA-21 expression in two human osteosarcoma cell lines. The reverse correlation between plasma lncRNA-NEF and miRNA-21 suggests the possibility of interactions between them. To further explore these potential interactions, lncRNA-NEF expression vectors and miRNA-21 mimics were transfected into the human osteosarcoma cell lines MG-63 and U2OS. IncRNA-NEF and miRNA-21 expression was subsequently detected using RT-qPCR (Fig. 4). Compared with the C and NC groups, lncRNA-NEF overexpression significantly decreased expression of miRNA-21 in the two cell lines $(\mathrm{P}<0.05$; Fig. $4 \mathrm{~A})$. By contrast, miRNA-21 overexpression resulted in no significant effects on the expression of lncRNA-NEF (P<0.05; Fig. 4B).

IncRNA-NEF overexpression inhibits migration of osteosarcoma cells, possibly through miRNA-21. Transwell migration and invasion assays were performed to investigate the effects of lncRNA-NEF and miRNA-21 overexpression on the migration and invasion abilities of MG-63 and U2OS human osteosarcoma cell lines. Compared with the $\mathrm{C}$, control miRNA and empty vector groups, IncRNA-NEF overexpression significantly inhibited and miRNA-21 overexpression significantly promoted migration $(\mathrm{P}<0.05$; Fig. $5 \mathrm{~A})$ and invasion $(\mathrm{P}<0.05$; Fig. 5B) of MG-63 and U2OS human osteosarcoma cell lines. 

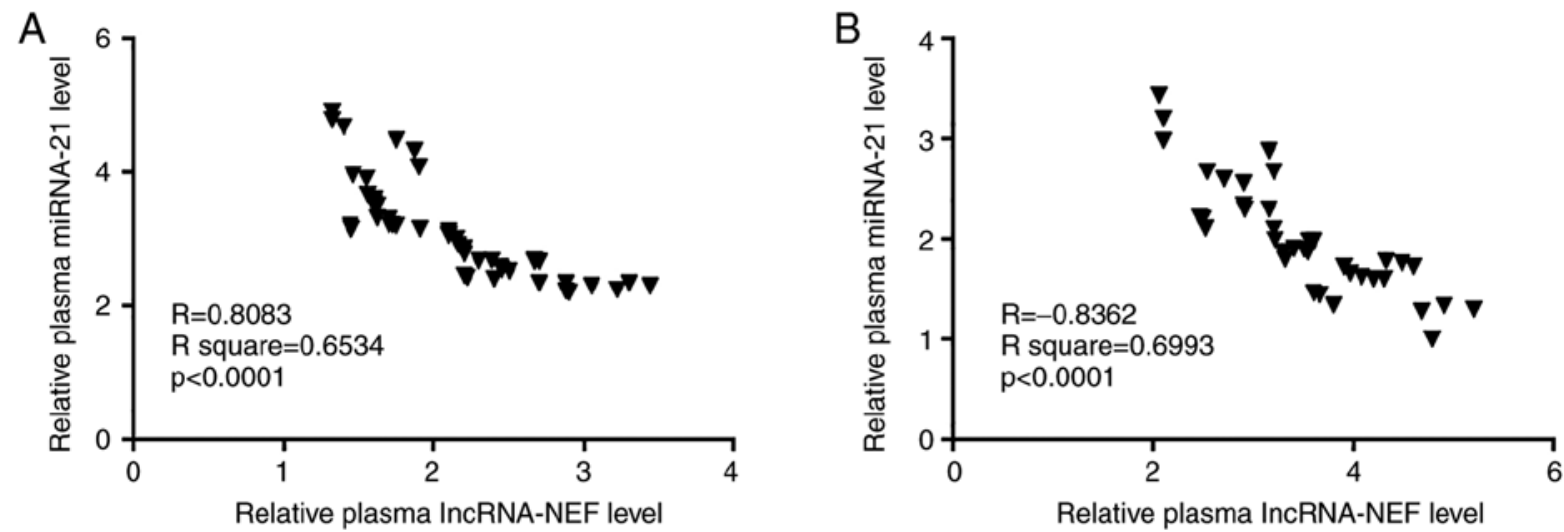

Figure 3. Plasma lncRNA-NEF and miRNA-21 are significantly and reversely correlated in patients with osteosarcoma and healthy controls. The Pearson's correlation test revealed that plasma lncRNA-NEF and miRNA-21 were significantly and reversely correlated in (A) patients with osteosarcoma and (B) healthy controls. miRNA, micro RNA; lncRNA, long non-coding RNA; NEF, neighboring enhancer of FOXA2.
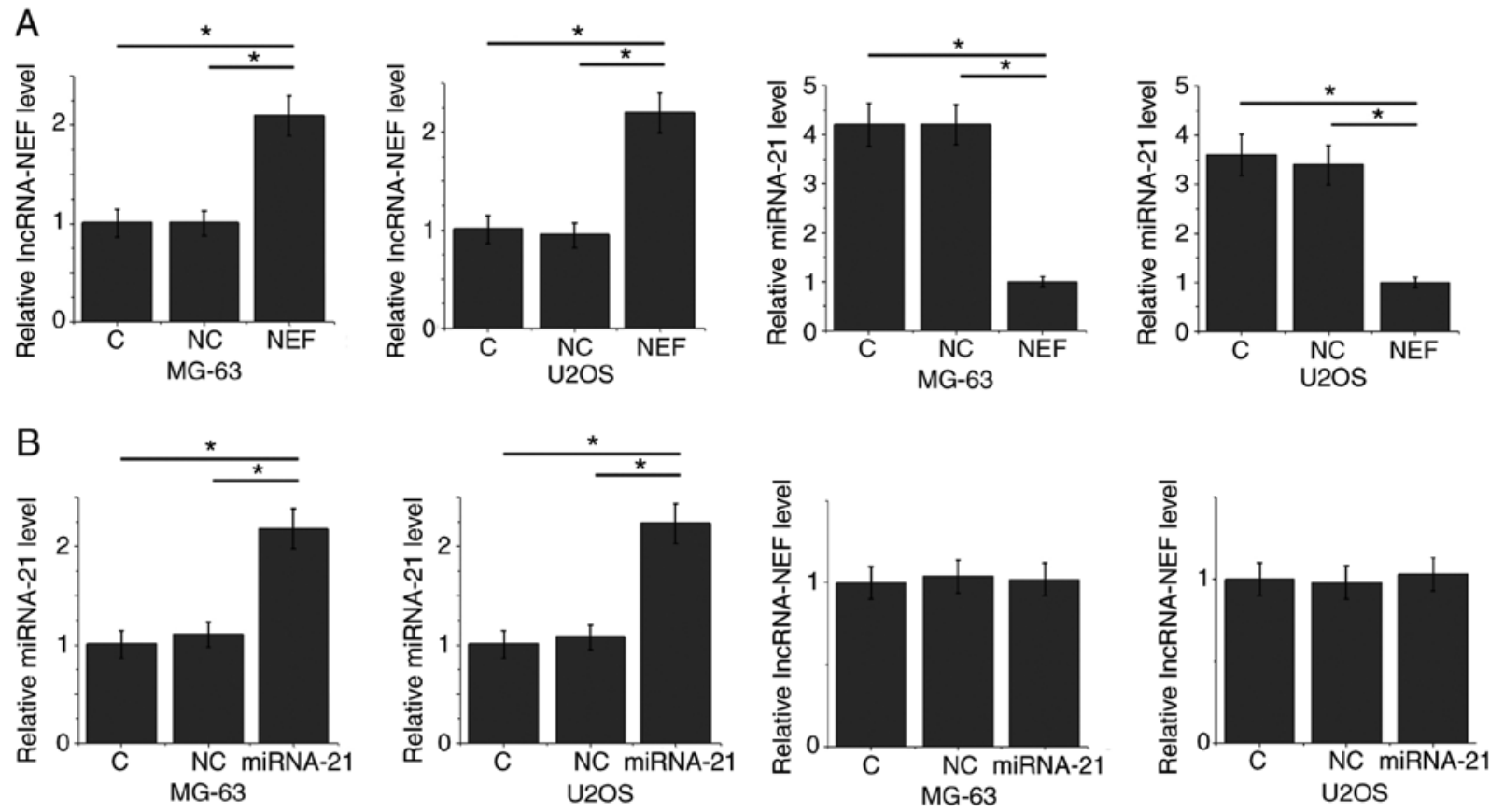

Figure 4. IncRNA-NEF overexpression leads to inhibited miRNA-21 expression in the human osteosarcoma cell lines MG-63 and U2OS. (A) lncRNA-NEF overexpression led to significantly inhibited expression of miRNA-21 in MG-63 and U2OS cells. (B) miRNA-21 overexpression revealed no significant effects on the expression of IncRNA-NEF in MG-63 and U2OS cells. "P<0.05. miRNA, micro RNA; lncRNA, long non-coding RNA; C, control; NC, negative control; NEF, neighboring enhancer of FOXA2.

Compared with cells with IncRNA-NEF overexpression alone, cells with IncRNA-NEF and miRNA-21 overexpression exhibited significantly reduced migration $(\mathrm{P}<0.05$; Fig. $5 \mathrm{~A})$ and invasion $(\mathrm{P}<0.05$; Fig. 5B) abilities.

\section{Discussion}

lncRNA-NEF is an oncogenic lncRNA which may inhibit epithelial to mesenchymal transition in hepatocellular carcinoma (13); however, its involvement in other human diseases is unknown. The present study revealed that lncRNA-NEF may inhibit cancer cell migration and invasion in osteosarcoma. These effects may be mediated by the downregulation of
miRNA-21, a well-characterized oncogenic miRNA in cancer biology (11).

Treatment of osteosarcoma is challenged by the high prevalence rate of tumor metastasis by the time of diagnosis (2). Therefore, early diagnosis is required to improve patient outcomes. The current study enrolled patients with stage I and II osteosarcoma, which are early stages of osteosarcoma. Downregulation of lncRNA-NEF was previously observed in patients with hepatocellular carcinoma (13). The present study revealed significantly decreased plasma levels of lncRNA-NEF in patients with osteosarcoma compared with healthy controls. Downregulation of plasma lncRNA-NEF effectively distinguished patients with osteosarcoma from 


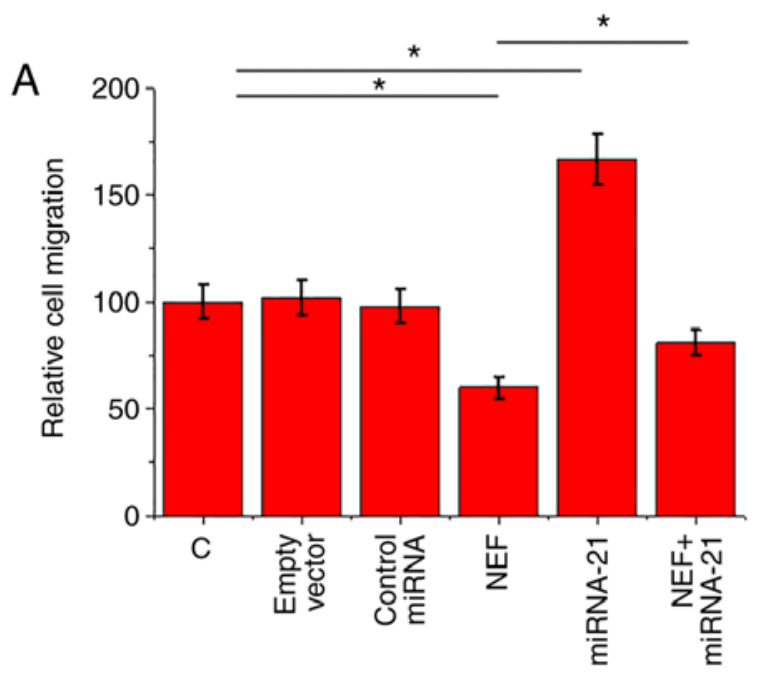

MG-63

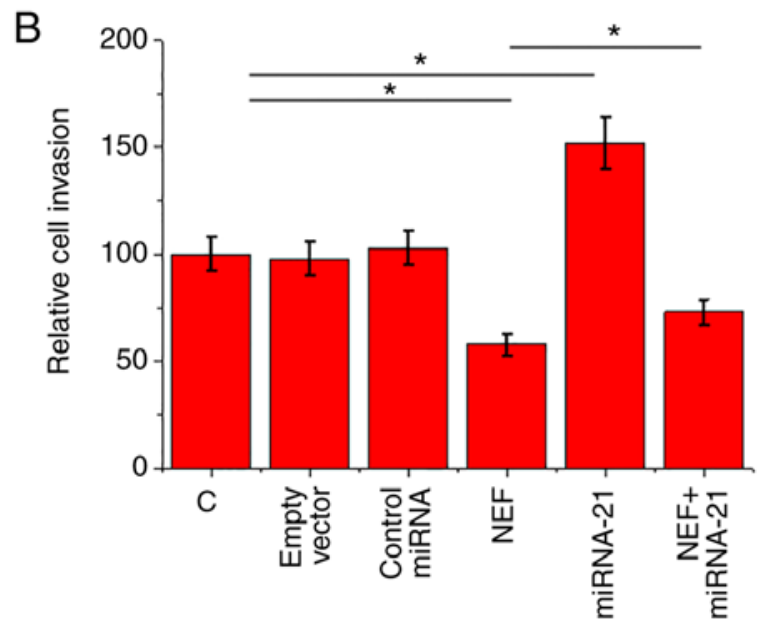

MG-63

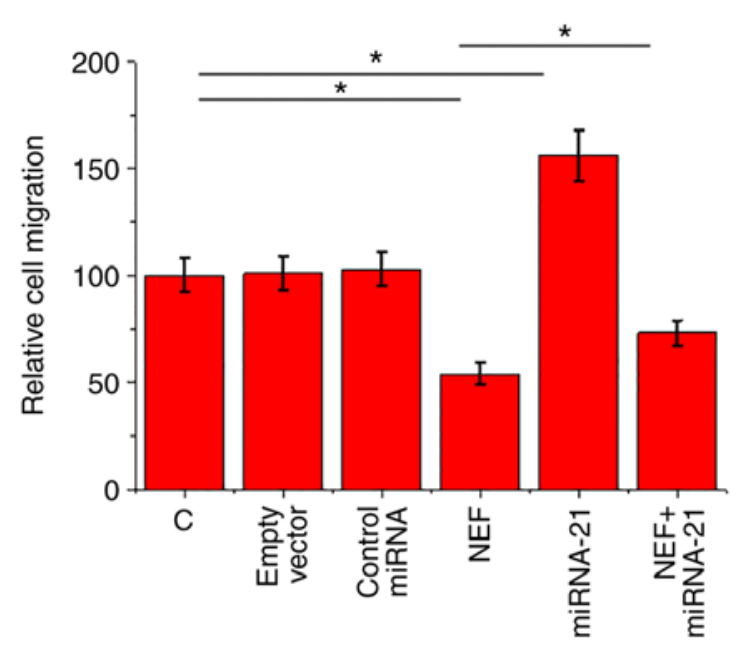

U2OS

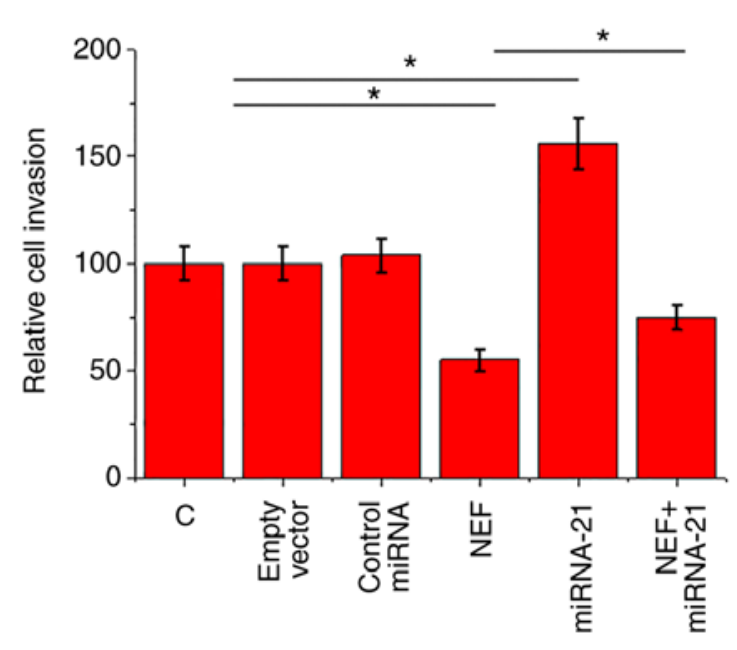

U2OS

Figure 5. IncRNA-NEF overexpression inhibits migration of the human osteosarcoma cell lines MG-63 and U2OS, possibly through miRNA-21. IncRNA-NEF overexpression significantly inhibited, while miRNA-21 overexpression significantly promoted (A) migration and (B) invasion of cells of MG-63 and U2OS cell lines. miRNA-21 overexpression attenuated the inhibitory effects of lncRNA-NEF overexpression on the migration and invasion of cancer cells. " $\mathrm{P}<0.05$. miRNA, micro RNA; lncRNA, long non-coding RNA; C, control; NEF, neighboring enhancer of FOXA2.

healthy controls, indicating that decreased levels of plasma IncRNA-NEF may have diagnostic value in the early detection of osteosarcoma. Subsequent in vitro experiments revealed that IncRNA-NEF may promote cancer cell invasion and migration in osteosarcoma. Therefore, the downregulation of IncRNA-NEF in patients with osteosarcoma may be used to predict tumor metastasis. IncRNA-NEF may serve as a therapeutic target for the prevention of tumor metastasis in osteosarcoma.

As an oncogenic miRNA, miRNA-21 is upregulated in different types of human malignancies, including osteosarcoma (15-17). Consistent with previous studies, the current study observed significantly upregulated plasma miRNA-21 in patients with osteosarcoma compared with healthy controls. Overexpression of miRNA-21 distinguished patients with osteosarcoma from healthy controls. However, altered expression of miRNA-21 has been observed in different types of cancer (11,15-17). Therefore, the diagnostic specificity of plasma miRNA-21 for osteosarcoma may be low.
The present study demonstrated a reverse correlation between plasma miRNA-21 and IncRNA-NEF in both patients with osteosarcoma and healthy controls. In vitro experiments using osteosarcoma cell lines revealed that lncRNA-NEF is a potential upstream inhibitor of miRNA-21 in the regulation of migration and invasion of osteosarcoma cells. 1ncRNA-NEF overexpression failed to significantly affect osteosarcoma cell proliferation (data not shown, revealed by Cell Counting Kit- 8 assay). miRNA-21 has been previously reported to be involved in the regulation of cancer proliferation in osteosarcoma (17-19). Therefore, lncRNA-NEF may antagonize the effects of downregulation of miRNA-21 on cancer cell proliferation. The current study reported that lncRNA-NEF may interact with a miRNA to participate in cancer development.

In conclusion, IncRNA-NEF is downregulated and miRNA is upregulated in patients with osteosarcoma compared with healthy controls. IncRNA-NEF may inhibit cancer cell migration and invasion in osteosarcoma by downregulating miRNA-21. 


\section{Acknowledgements}

Not applicable.

\section{Funding}

No funding was received.

\section{Availability of data and materials}

The datasets used and/or analyzed during the present study are available from the corresponding author on reasonable request.

\section{Authors' contributions}

QLY, HY, XH, QSY and CZ performed the experiments. QLY analyzed the data and was a major contributor in writing the manuscript. All authors read and approved the final manuscript.

\section{Ethics approval and consent to participate}

Ethical approval was obtained from the Ethics Committee of Guangzhou General Hospital of Guangzhou Military Command. The study followed the tenets of the Declaration of Helsinki, and written informed consent was obtained from all patients and controls after the nature and possible consequences of the study were explained.

\section{Patient consent for publication}

Not applicable.

\section{Competing interests}

The authors declare that they have no competing interests.

\section{References}

1. Ottaviani G and Jaffe N: The epidemiology of osteosarcoma. Cancer Treat Res 152: 3-13, 2009.

2. Marko TA, Diessner BJ and Spector LG: Prevalence of metastasis at diagnosis of osteosarcoma: An international comparison. Pediatr Blood Cancer 63: 1006-1011, 2016.

3. Luetke A, Meyers PA, Lewis I and Juergens H: Osteosarcoma treatment-where do we stand? A state of the art review. Cancer Treat Rev 40: 523-532, 2014.

4. Li JZ, Tian ZQ, Jiang SN and Feng T: Effect of variation of ABCB1 and GSTP1 on osteosarcoma survival after chemotherapy. Genet Mol Res 13: 3186-3192, 2014.

5. Anderson ME: Update on survival in osteosarcoma. Orthop Clin North Am 47: 283-292, 2016.
6. Denduluri SK, Wang Z, Yan Z, Wang J, Wei Q, Mohammed MK, Haydon RC, Luu HH and He TC: Molecular pathogenesis and therapeutic strategies of human osteosarcoma. J Biomed Res 30: 2015.

7. Mattick JS and Makunin IV: Non-coding RNA. Hum Mol Genet 15: R17-R29, 2006.

8. Eddy SR: Non-coding RNA genes and the modern RNA world. Nat Rev Genet 2: 919-929, 2001.

9. Esteller M: Non-coding RNAs in human disease. Nat Rev Genet 12: 861-874, 2011.

10. Taft RJ, Pang KC, Mercer TR, Dinger M and Mattick JS: Non-coding RNAs: Regulators of disease. J Pathol 220: 126-139, 2010.

11. Kumarswamy R, Volkmann I and Thum T: Regulation and function of miRNA-21 in health and disease. RNA Biol 8: 706-713, 2011.

12. Dan J, Wang J, Wang Y, Zhu M, Yang X, Peng Z, Jiang $H$, and Chen L: LncRNA-MEG3 inhibits proliferation and metastasis by regulating miRNA-21 in gastric cancer. Biomed Pharmacother 99: 931-938, 2018.

13. Liang WC, Ren JL, Wong CW, Chan SO, Waye MM, Fu WM and Zhang JF: LncRNA-NEF antagonized epithelial to mesenchymal transition and cancer metastasis via cis-regulating FOXA2 and inactivating Wnt/beta-catenin signaling. Oncogene 37: 1445-1456, 2018.

14. Livak KJ and Schmittgen TD: Analysis of relative gene expression data using real-time quantitative PCR and the 2(-Delta Delta C(T)) method. Methods 25: 402-408, 2001.

15. Han Y, Xu GX, Lu H, Yu DH, Ren Y, Wang L, Huang XH, Hou WJ, Wei ZH, Chen YP, et al: Dysregulation of miRNA-21 and their potential as biomarkers for the diagnosis of cervical cancer. Int J Clin Exp Pathol 8: 7131-7139, 2015.

16. Costa PM, Cardoso AL, Custodia C, Cunha P, Pereira de Almeida L and Pedroso de Lima MC: MiRNA-21 silencing mediated by tumor-targeted nanoparticles combined with sunitinib: A new multimodal gene therapy approach for glioblastoma. J Control Release 207: 31-39, 2015.

17. Zhang QL, Yang SH, Liu HY and Wu CH: Nhibition of osteosarcoma cell proliferation by a short hairpin RNA targeting proliferation cell nuclear antigen. Zhonghua Bing Li Xue Za Zhi 34: 167-170, 2005 (In Chinese).

18. Li C, Xu B, Miu X, Deng Z, Liao H and Hao L: Inhibition of miRNA-21 attenuates the proliferation and metastasis of human osteosarcoma by upregulating PTEN. Exp Ther Med 15: 1036-1040, 2018

19. Zhao J, Zhang C, Gao Z, Wu H, Gu R and Jiang R: Long non-coding RNA ASBEL promotes osteosarcoma cell proliferation, migration, and invasion by regulating microRNA-21. J Cell Biochem 119: 6461-6469, 2018.

This work is licensed under a Creative Commons Attribution-NonCommercial-NoDerivatives 4.0 International (CC BY-NC-ND 4.0) License. 